\title{
SONDAGEM ELÉTRICA VERTICAL APLICADA EM PESQUISA HIDROGEOLÓGICA NA BACIA DO PARECIS, MT
}

\author{
Alterêdo Oliveira Cutrim ${ }^{1}$, Amarildo Salina Ruiz², Lucas Matos Liporoni ${ }^{3}$, Felipe A. de Medeiros ${ }^{4}$, \\ Uendel Chaves Barroso ${ }^{5}$ e Alexandrino L. Nascimento ${ }^{6}$ \\ Recebido em 4 dezembro, 2006 / Aceito em 28 junho, 2007 \\ Received on December 4, 2006 / Accepted on June 28, 2007
}

\begin{abstract}
The research was carried out in Lucas do Rio Verde city, Mato Grosso State, Brazil, to estimate the depth and thickness of hydrogeological units. This parameter was estimated using vertical electrical sounding (VES), with AB/2 maximum of $2000 \mathrm{~m}$. The results show that the average depth and thickness of Utiariti aquifer are respectively $13 \mathrm{~m}$ and $90 \mathrm{~m}$, the Saltos das Nuvens Formation has average depth of $103 \mathrm{~m}$ and thickness greater than $600 \mathrm{~m}$. Only the portion of the Utiariti aquifer consisted essentially of fine to medium sandstone was defined very precisely by the VESs. Geological data from the well, used in the interpretation of VES allowed greater reliability on the obtained results.
\end{abstract}

Keywords: VES, Parecis Basin, hydrogeology.

RESUMO. Esta pesquisa foi realizada na cidade de Lucas do Rio Verde, estado de Mato Grosso, e teve como objetivo estimar a profundidade e a espessura das unidades hidrogeológicas para subsidiar pesquisa hidrogeológica nessa área. Para tanto foi aplicada a técnica da Sondagem Elétrica Vertical (SEV), com abertura máxima de AB/2 de $2000 \mathrm{~m}$. Os resultados das SEVs mostram que o Aqǘffero Utiariti tem profundidade ao topo e espessura média de $13 \mathrm{~m}$ e $90 \mathrm{~m}$ respectivamente, e a Formação Salto das Nuvens tem profundidade ao topo média de $103 \mathrm{~m}$ e espessura superior a $600 \mathrm{~m}$. Somente a parte do Aquífero Utiariti, constituída essencialmente por arenitos de granulação fina e média foi evidenciada com muita precisão nas curvas das SEVs. Os dados do perfil geológico do poço, usados na interpretação das SEVs, possibilitaram maior confiabilidade nos resultados obtidos.

Palavras-chave: SEV, Bacia do Parecis, hidrogeologia.

\footnotetext{
${ }^{1}$ Universidade Federal de Mato Grosso, UFMT, Instituto de Ciências Exatas e da Terra, ICET, Av. Fernando Correa da Costa, s/n, Coxipó - 78060-900 Cuiabá, MT, Brasil. Tel.: (65) 3615-8751; Fax: (65) 3615-8752 - E-mail: alteredo@ufmt.br

2 Universidade Federal de Mato Grosso, UFMT, Instituto de Ciências Exatas e da Terra, ICET, Av. Fernando Correa da Costa, s/n, Coxipó - 78060-900 Cuiabá, MT, Brasil. Tel.: (65) 3615-8751; Fax: (65) 3615-8752 -E-mail: asruiz@ufmt.br

3 Universidade Federal de Mato Grosso, UFMT, Faculdade de Arquitetura e Engenharia, FAET, Av. Fernando Correa da Costa, s/n, Coxipó - 78060-900 Cuiabá, MT, Brasil. E-mail: lucas_liporoni@hotmail.com

${ }^{4}$ Universidade Federal de Mato Grosso, UFMT, Instituto de Ciências Exatas e da Terra, ICET, Av. Fernando Correa da Costa, s/n, Coxipó - 78060-900 Cuiabá, MT, Brasil. Tel.: (65) 3615-8751; Fax: (65) 3615-8752 - E-mail: xaketu@hotmail.com

5 Universidade Federal de Mato Grosso, UFMT, Instituto de Ciências Exatas e da Terra, ICET, Av. Fernando Correa da Costa, s/n, Coxipó - 78060-900 Cuiabá, MT, Brasil. Tel.: (65) 3615-8751; Fax: (65) 3615-8752 - E-mail: barrosoge|@gmail.com

6 Universidade Federal de Mato Grosso, UFMT, Instituto de Ciências Exatas e da Terra, ICET, Av. Fernando Correa da Costa, s/n, Coxipó - 78060-900 Cuiabá, MT, Brasil. Tel.: (65) 3615-8751; Fax: (65) 3615-8752 -E-mail: leite@ufmt.br
} 


\section{INTRODUÇÃo}

A pesquisa hidrogeológica, envolvendo a estimativa de reservas de água, a avaliação de vulnerabilidade à contaminação de aqüífero, a formulação de modelo hidrogeológico conceitual e a elaboração de projeto de construção de poço tubular, necessita do conhecimento da espessura e da profundidade de unidades hidrogeológicas. As reservas de água de um aqüífero são diretamente proporcionais à sua espessura (Fetter, 2002; Custódio \& Llamas, 2002; Costa, 2000; Cutrim \& Rebouças, 2005a), assim como a profundidade ao topo do aqüífero é um dos parâmetros utilizados em estudo de vulnerabilidade à contaminação de aqüífero, dada a sua influência no tempo de trânsito dos contaminantes (Foster et al., 2002; Aller et al., 1987). A espessura e a profundidade ao topo são utilizadas na elaboração de projeto de construção de poços tubulares profundos (Custódio \& Llamas, 2002; Fetter, 2002), visto que elas permitem estimar a profundidade do poço a qual tem influência direta nos custos de perfuração e na indicação do porte da máquina de perfuração. Na formulação de modelo hidrogeológico conceitual, estes parâmetros constituem as dimensões verticais do modelo (Anderson \& Woessner, 1992).

Ressalta-se que a precisão da estimativa das reservas de um aqüífero, a veracidade do seu modelo hidrogeológico conceitual e um bom projeto de poço tubular, dependem de um bom conhecimento da distribuição, espessura e profundidade das unidades hidrogeológicas, o que na maioria dos casos, inexistem ou são raras e muito imprecisas, como é o caso da área desta pesquisa, onde o conhecimento hidrogeológico está apenas em nível preliminar, tendo apenas um poço tubular de $150 \mathrm{~m}$ de profundidade. Além disso, na área está prevista a construção de poços tubulares profundos para captar em torno de $19.000 \mathrm{~m}^{3}$ de água por dia.

Esse cenário mostra a grande necessidade do conhecimento da espessura e da profundidade das unidades hidrogeológicas da área para viabilizar um maior conhecimento dessas águas subterrâneas e orientar a construção de poços tubulares profundos. Como a área está inserida na Bacia sedimentar do Parecis, então foi aplicada a técnica da Sondagem Elétrica Vertical - SEV, dada a sua reconhecida eficiência na estimativa desses parâmetros, nesse tipo de geologia (Cutrim \& Rebouças, 2005b; Cutrim \& Fachin, 2004; Cutrim et al., 2002; Cutrim et al., 2001). Para aumentar o grau de precisão da estimativa desses parâmetros, o modelo interpretativo das SEVs foi postulado com auxilio das informações geológicas disponíveis da área, incluindo aquelas do perfil geológico do poço tubular.

\section{CARACTERÍSTICAS DA ÁREA DE ESTUDO Aspectos gerais}

A área de pesquisa localiza-se no perímetro urbano da cidade de Lucas do Rio Verde, situada no centro-norte do estado de
Mato Grosso (Fig. 1). A cidade de Lucas do Rio Verde tem uma população em torno de 40.000 habitantes, e é uma cidade com grande índice de desenvolvimento social e econômico, onde a base desse desenvolvimento é a agricultura e a pecuária, sendo 0 município o maior produtor de suínos do estado.

Todo esse desenvolvimento tem o seu planejamento pautado na água subterrânea como a principal fonte de abastecimento de água. No entanto, o nível de conhecimento sobre essas águas, em todos os aspectos, é muito pequeno ou inexistente.

\section{Geologia da área}

A área estudada está inserida na Bacia do Parecis (Barros et al., 1982), no entanto é marcada pela homogeneidade em sua constituição geológica, sendo notável a vasta exposição de Latossolo vermelho em quase a totalidade de sua extensão, sendo raríssimas as exposições de arenitos vermelhos ferruginosos, carapaças lateríticas e cataclasitos nas margens e leito do rio Verde.

Devido à profundidade de investigação envolver apenas as unidades estratigráficas Formações Salto das Nuvens e Utiariti, então somente estas foram descritas a seguir.

A Formação Salto das Nuvens, na parte de topo, é constituído por arenitos finos, siltitos e níveis argilosos, consolidados a semiconsolidados, e por argilito e siltito nas suas demais porções.

A Formação Utiariti, sobreposta à Formação Salto das Nuvens, raramente exposta nas margens do rio Verde, é constituída por arenitos quartzo-feldspáticos, de granulação fina a média, de cor vermelha a marrom, intensamente intemperizados.

Com base na seção colunar do poço tubular (Fig. 2) notase que, em subsuperfície, a Formação Utiariti é constituída por uma intercalação de arenitos quartzosos, brancos, de granulação fina a média, arenitos vermelhos finos, com intercalações de siltitos e argilitos e arenitos vermelhos de granulação média a grossa. Esta formação tem uma espessura em torno de $135 \mathrm{~m}$, sendo $50 \mathrm{~m}$ de arenitos quartzosos de cor branca, granulação fina; $45 \mathrm{~m}$ de arenito vermelho de textura média e os demais $40 \mathrm{~m}$ são as intercalações de arenitos argilosos e arenitos de granulação grossa.

A superfície paleogênica peneplanizada com latossolização (PRODEAGRO, 2001) corresponde a quase totalidade da área mapeada, sendo essencialmente composta por vastas ocorrências de Latossolos vermelhos e crostas lateríticas subordinadas.

As crostas lateríticas foram observadas nas proximidades do rio Verde, em discretas quebras topográficas ocasionadas pela erosão remontante. Formam bancos concrecionários métricos compostos por lateritos ferruginosos, com aspecto botroidal, com grânulos e grãos de quartzo leitoso e fragmentos líticos. 


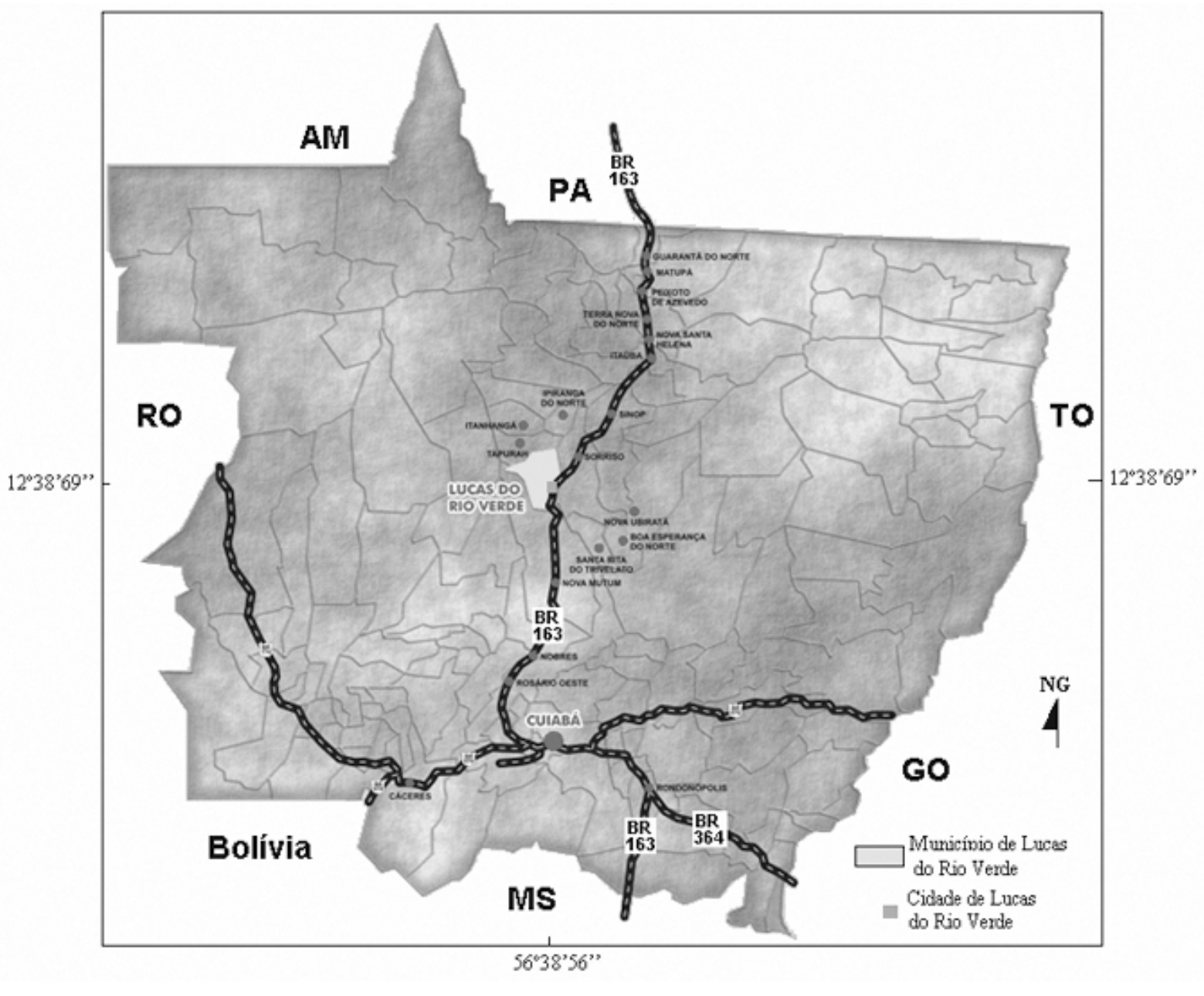

Figura 1 - Mapa de localização da área de estudo.

0 Latossolo vermelho-escuro recobre quase a totalidade da área investigada. Trata-se de um solo muito intemperizado, profundo, com grande homogeneidade, textura argilosa, plástico e ligeiramente pegajoso.

As aluviões atuais estão restritas à calha do rio Verde e são compostas essencialmente por areias finas a médias, quartzosos de cor branco-amarelada com subordinada proporção de argilas.

\section{Hidrogeologia da área}

Na área, o Aqǘfero Utiariti constitui o principal reservatório de água subterrânea, é um aqüifero livre, onde existem doze poços com profundidade de 35 a $40 \mathrm{~m}$, produzindo vazões de 5 a $6 \mathrm{~m}^{3} / \mathrm{h}$, com nível dinâmico variando de $18 \mathrm{~m}$ a $25 \mathrm{~m}$. 0 único poço mais profundo da área (profundidade de $150 \mathrm{~m}$ ) produz vazão de 100 m³/h com nível dinâmico de $53 \mathrm{~m}$.

\section{METODOLOGIA}

Nesta pesquisa foi aplicada a técnica da Sondagem Elétrica Vertical - SEV, a qual permite investigar a variação vertical de resistividade. Consiste em injetar corrente elétrica no meio através de dois pontos ( $A$ e B) e medir a diferença de potencial entre dois outros pontos ( $\mathrm{M}$ e N) localizados entre os pontos de corrente (Fig. 3). Através da corrente, da diferença de potencial e do fator geométrico (função das distâncias entre os pontos de injeção de corrente e de medidas de potencial) determina-se a resistividade aparente do meio, através da equação apresentada em Bhattacharya \& Patra (1986):

$$
\begin{aligned}
\rho_{a} & =\left(\frac{\Delta V}{I}\right) k \\
k & =\frac{2 \pi}{\frac{1}{A M}-\frac{1}{B M}-\frac{1}{A N}+\frac{1}{B N}}
\end{aligned}
$$


onde:

$\rho_{a}=$ resistividade aparente;

$\Delta V=$ diferença de potencial;

$I \quad=$ corrente elétrica;

$A M=$ distância entre os eletrodos $A$ e $M$;

$A N=$ distância entre os eletrodos $A$ e $N$;

$B M=$ distância entre os eletrodos $B$ e $M ; \mathrm{e}$

$B N=$ distância entre os eletrodos $B$ e $N$.

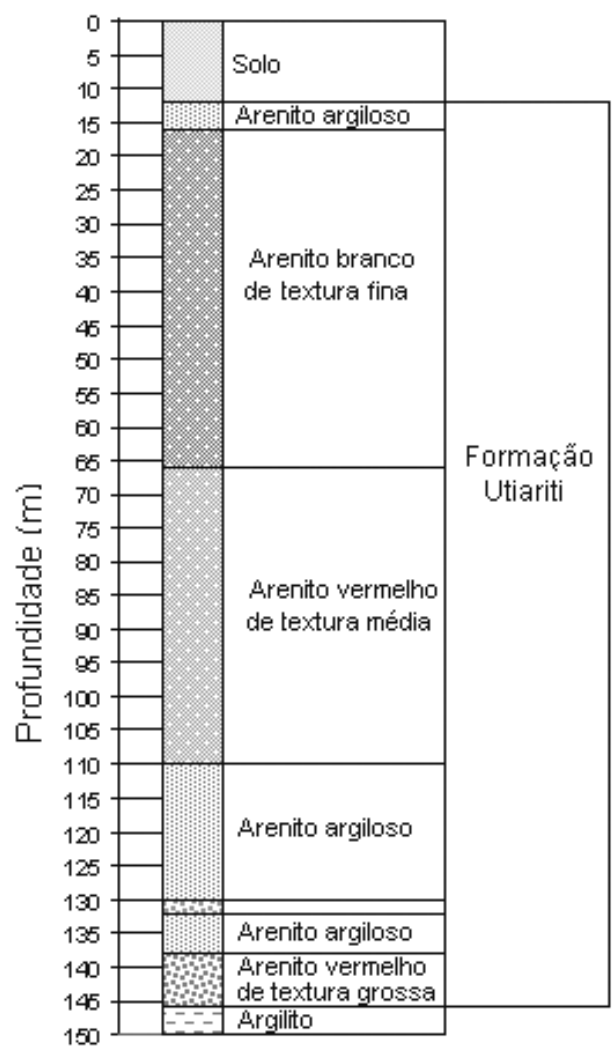

Figura 2 - Perfil geológico do poço tubular profundo da área.

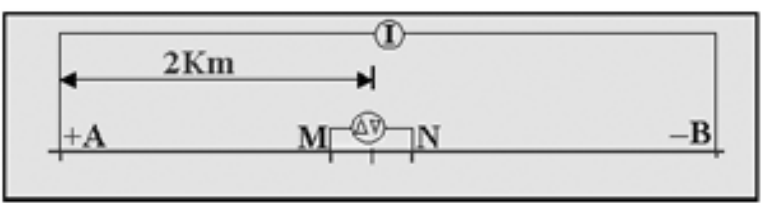

Figura 3 - Arranjo Schlumberger.

Como a corrente flui de modo radial, então quanto maior a distância entre os pontos de injeção de corrente, maior será a profundidade investigada.

0 equipamento usado para coletar os dados foi um resistivímetro, com potência máxima de $250 \mathrm{~W}$ e voltagens de $100 \mathrm{~V}$, 200 V, 400 V e 800 V. A resistência de contato nos eletrodos foi minimizada com 0 uso de água salgada. Foram realizadas nove
SEVs, com abertura máxima de $\mathrm{AB} / 2$ de $2 \mathrm{~km}$ e a distância de $\mathrm{MN} \leq \mathrm{AB} / 5$, distribuídas de modo a fornecer o melhor conhecimento da geologia da área, visando subsidiar estudos hidrogeológicos na área (Fig. 4).

As SEVs foram interpretadas através do método Ridge Regression (Tikhonov \& Arsenin, 1977), utilizando-se o software IPIwin2. Nesta etapa foram determinadas a profundidade ao topo e a espessura das unidades geológicas da área e as suas respectivas resistividades. No processo de interpretação das SEVs, a espessura das unidades geológicas apresentadas no perfil geológico do poço da Figura 2 foi usada para auxiliar a identificação dessas unidades nas curvas das SEVs, o que permitiu determinar com mais segurança a relação (AB/2) / espessura da unidade geológica, na determinação do modelo interpretativo das SEVs. Além disso, a relação (AB/2) / espessura da unidade geológica foi vinculada ao intervalo $(A B / 4, A B / 6)$.

\section{RESULTADOS E DISCUSSÕES}

Foram realizadas nove SEVs e os seus resultados estão apresentados nas Figuras 5 a 15 e na Tabela 1 . Todas as SEVs foram ajustadas usando um modelo de seis camadas para permitir o melhor ajuste possível.

Em todas as SEVs, as três primeiras camadas correspondem à cobertura pedológica, com espessura variando de $11,5 \mathrm{~m}$ a $15,6 \mathrm{~m}$ e resistividade com valores de $210 \Omega$.m a $1.510 \Omega . \mathrm{m}$. Os valores mais elevados de resistividade ocorrem sempre na segunda camada, correspondendo a uma camada de laterita, cuja espessura varia de $0,3 \mathrm{~m}$ a 2,7 $\mathrm{m}$.

A camada 4, em todas as SEVs, corresponde à Formação Utiariti, com profundidade ao topo de 11,5 m a 15,6 m, espessura variando de $75 \mathrm{~m}$ a $106 \mathrm{~m}$ e resistividade variando de $2.950 \Omega . \mathrm{m}$ a $4.700 \Omega$.m. Essa espessura estimada para esta formação corresponde apenas aos seus arenitos brancos e vermelhos, o que indica que os seus últimos $30 \mathrm{~m}$, constituídos por arenitos argilosos intercalados com finas camadas de arenito de textura grossa (Fig. 2) não foram evidenciados nas SEVs, provavelmente devido à pequena espessura dessas camadas.

As camadas 5 e 6, em todas as SEVs, referem-se à Formação Salto das Nuvens, cuja espessura não foi estimada por se tratar das últimas camadas (substrato) das SEVs. A resistividade dessa formação tem valores máximo e mínimo, respectivamente, de $243 \Omega$.m e $20 \Omega$.m.

Nota-se na parte final das SEVs, com AB/2 de 2000 m, que há uma tendência de ascensão da curva de resistividade, indicando provavelmente 0 fim da Formação Salto das Nuvens. 


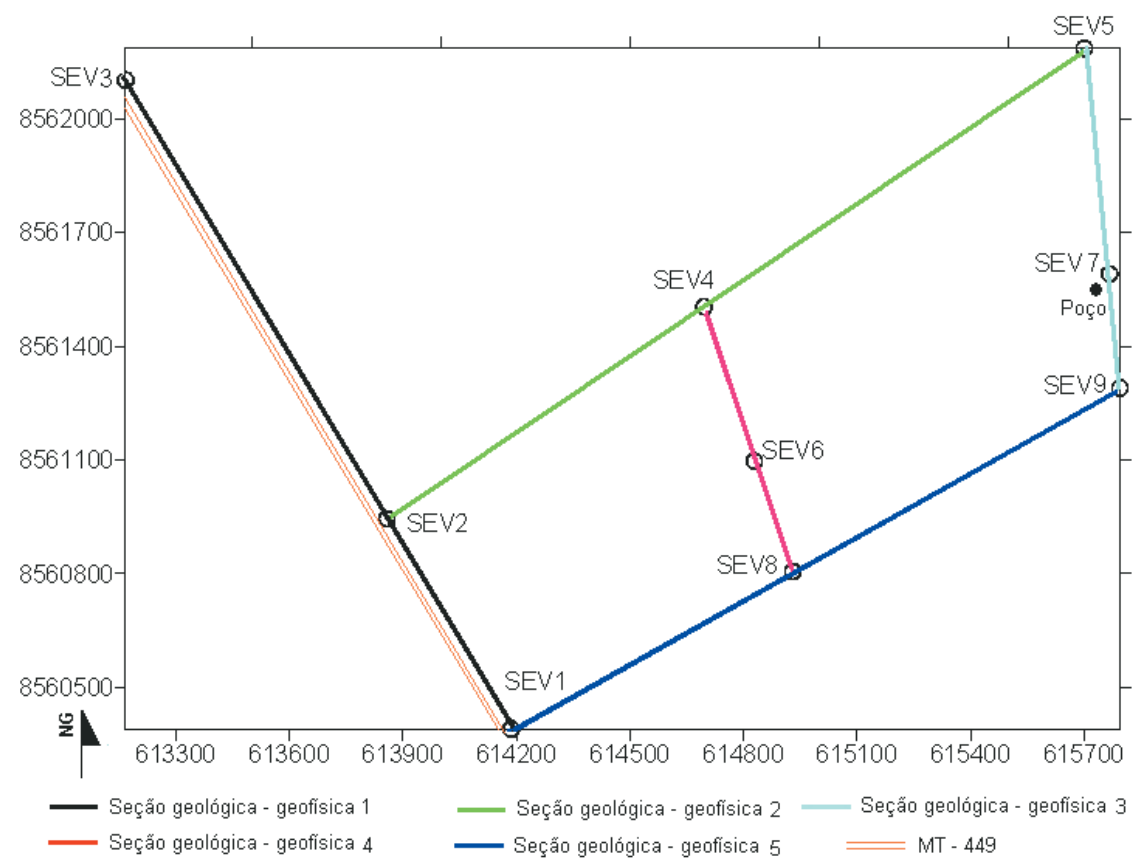

Figura 4 - Mapa de localização das SEVs e do poço.

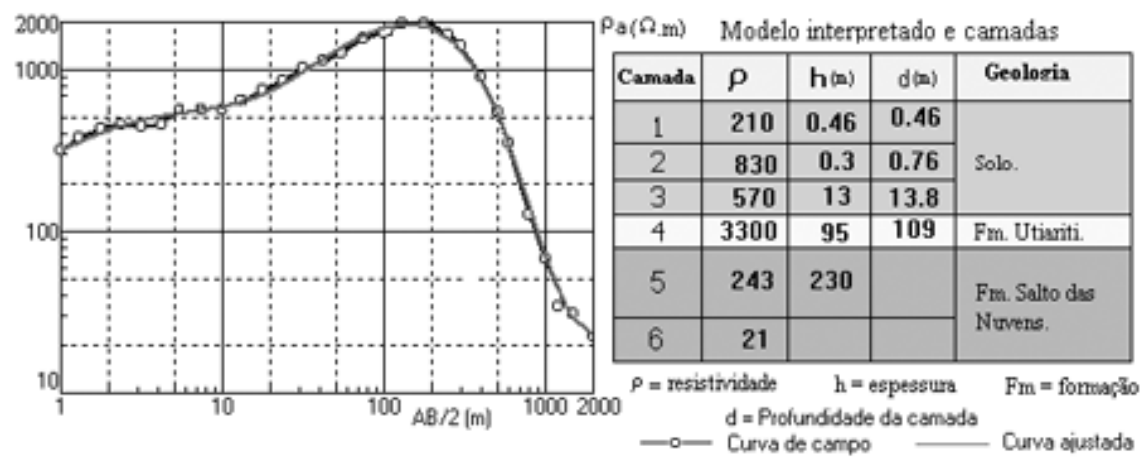

Figura 5 - Perfil geoelétrico da SEV1 e modelo interpretado.

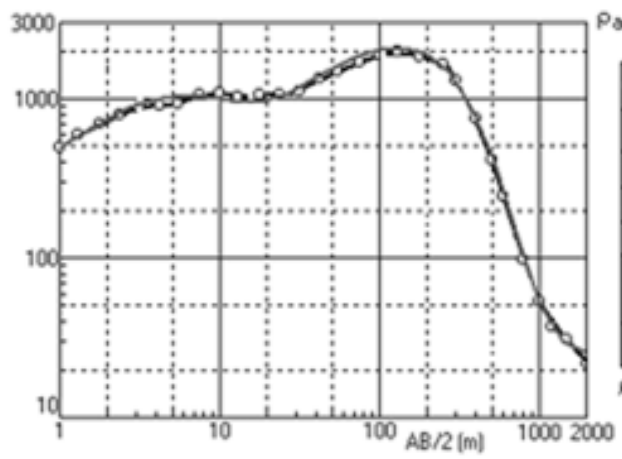

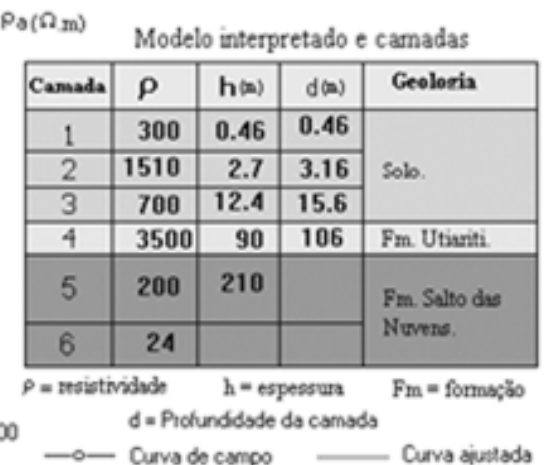

Figura 6 - Perfil geoelétrico da SEV2 e modelo interpretado. 


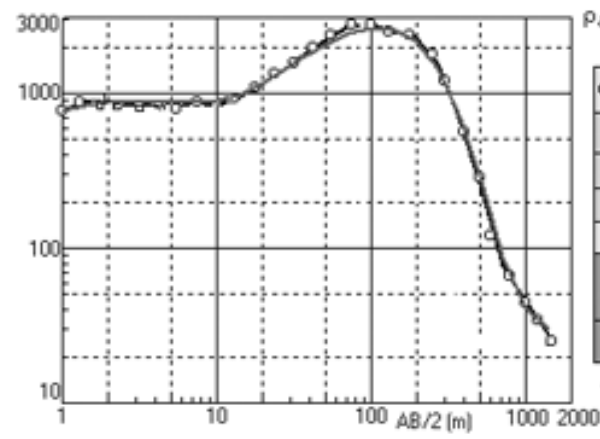

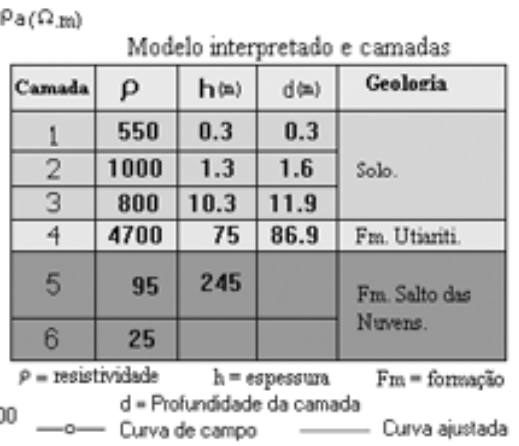

Figura 7 - Perfil geoelétrico da SEV3 e modelo interpretado.

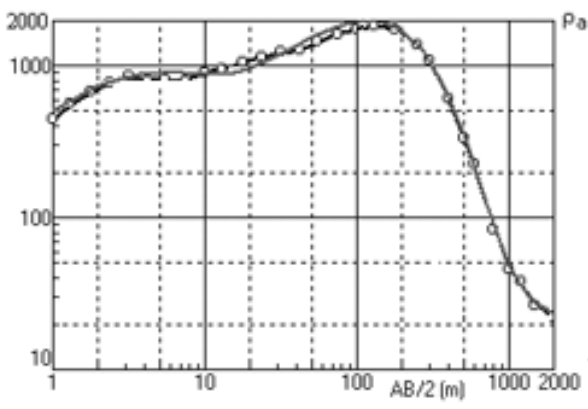

\begin{tabular}{|c|c|c|c|c|}
\hline$\left(\Omega_{\mathrm{m}} \mathrm{m}\right)$ & \multicolumn{4}{|c|}{ Modelo interpretado e camadas } \\
\hline Camada & $\rho$ & $h(\omega)$ & $d(m)$ & Geolozia \\
\hline 1 & 320 & 0.48 & 0.48 & \multirow{3}{*}{ Sob. } \\
\hline 2 & 1500 & 1.2 & 1.68 & \\
\hline 3 & 760 & 13 & 14.7 & \\
\hline 4 & 3100 & 90 & 105 & Fm. Utianti. \\
\hline 5 & 190 & 227 & & \multirow{2}{*}{$\begin{array}{l}\text { Fm. Salto das } \\
\text { Nuvere. }\end{array}$} \\
\hline 6 & 22 & & & \\
\hline \multicolumn{2}{|c|}{$\rho=$ resistividede } & \multirow{2}{*}{\multicolumn{2}{|c|}{$\begin{array}{l}\mathrm{h}=\text { espessura } \\
\text { fundidade da camada } \\
\text { de campo }\end{array}$}} & Fm $=$ fonmogio \\
\hline$-0-$ & $\begin{array}{l}\text { de Plod } \\
\text { Cunvad }\end{array}$ & & & - Curva ajustada \\
\hline
\end{tabular}

Figura 8 - Perfil geoelétrico da SEV4 e modelo interpretado.

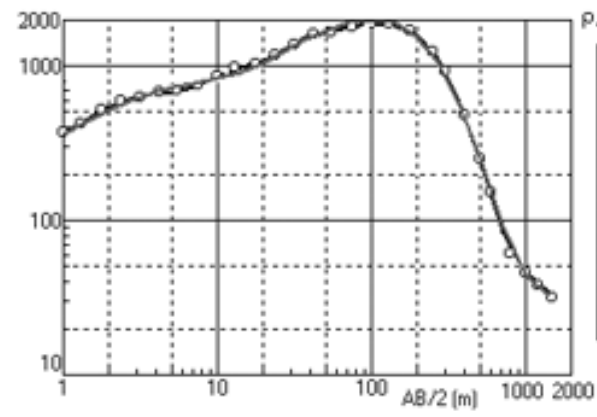

\begin{tabular}{|c|c|c|c|c|}
\hline$a\left(\Omega_{m}\right)$ & Mode & lo inter & retado & camadas \\
\hline Camada & $\rho$ & $h(\mathbf{m})$ & $d(\mathbf{a})$ & Geoloria \\
\hline 1 & 250 & 0.56 & 0.56 & \multirow{3}{*}{ Solo. } \\
\hline 2 & 1200 & 0.5 & 1.06 & \\
\hline 3 & 810 & 10.4 & 11.5 & \\
\hline 4 & 2950 & 82 & 93.5 & Fm. Utianiti. \\
\hline 5 & 210 & 200 & & \multirow{2}{*}{$\begin{array}{l}\text { Fm. Salto dis } \\
\text { Nurvens. }\end{array}$} \\
\hline 6 & 28 & & & \\
\hline & & \multicolumn{2}{|c|}{$\begin{array}{l}\mathrm{h}=\text { espessura } \\
\text { didade da camad }\end{array}$} & do $F m=$ formo \\
\hline
\end{tabular}

Figura 9 - Perfil geoelétrico da SEV5 e modelo interpretado.

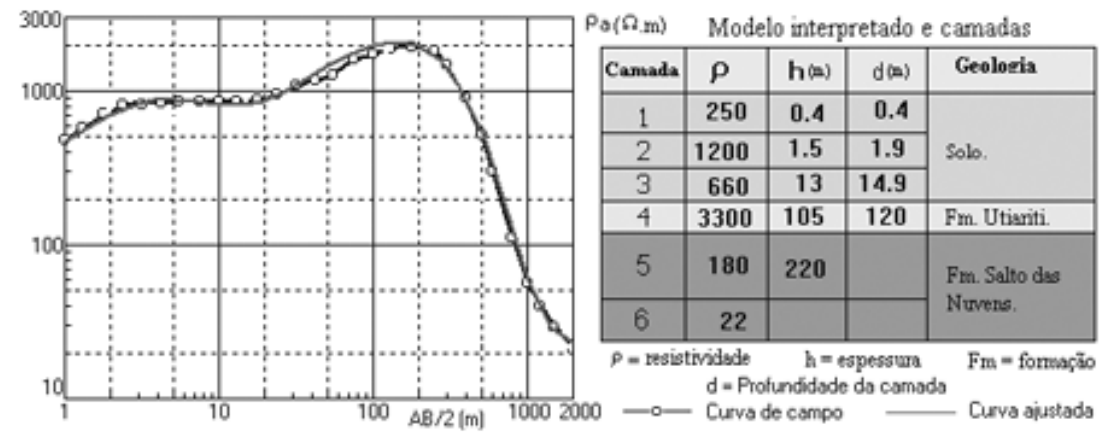

Figura 10 - Perfil geoelétrico da SEV6 e modelo interpretado. 


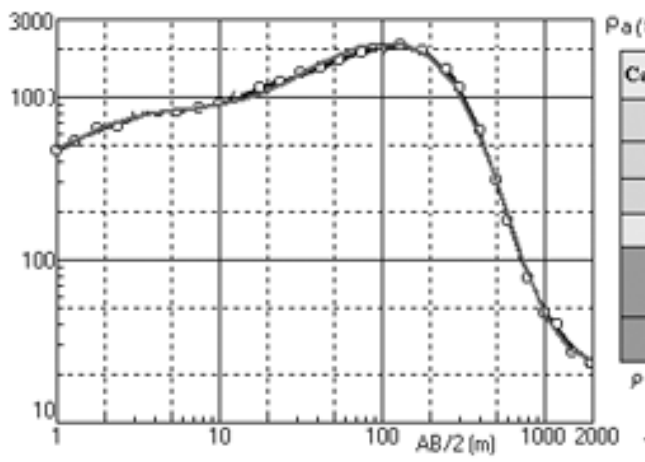

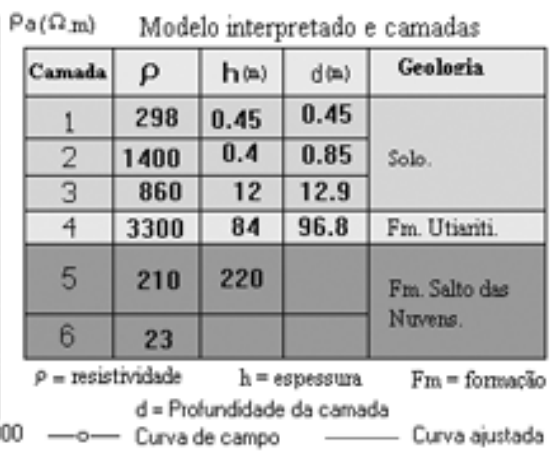

Figura 11 - Perfil geoelétrico da SEV7 e modelo interpretado.

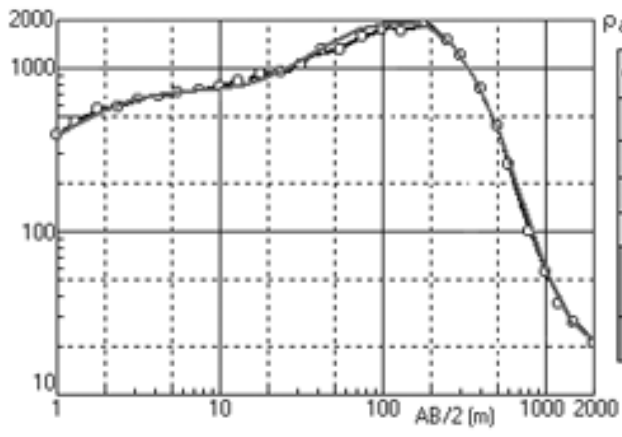

\begin{tabular}{|c|c|c|c|c|}
\hline$a\left(\Omega_{m}\right)$ & Mod & do inter & retado & camadas \\
\hline Camada & $\rho$ & $h(n)$ & $d(n)$ & Geoloria \\
\hline 1 & 250 & 0.46 & 0.46 & \multirow{3}{*}{ Solo. } \\
\hline 2 & 1000 & 1 & 1.46 & \\
\hline 3 & 710 & 13 & 14.5 & \\
\hline 4 & 3000 & 106 & 109 & Fm. Utianiti. \\
\hline 5 & 150 & 240 & & \multirow{2}{*}{$\begin{array}{l}\text { Fm. Salto das } \\
\text { Nuvens. }\end{array}$} \\
\hline 6 & 20 & & & \\
\hline \multicolumn{2}{|c|}{$\rho=$ resistividade } & $\mathrm{h}=$ & pessura & $\mathrm{Fm}=$ fonmo \\
\hline
\end{tabular}

Figura 12 - Perfil geoelétrico da SEV8 e modelo interpretado.
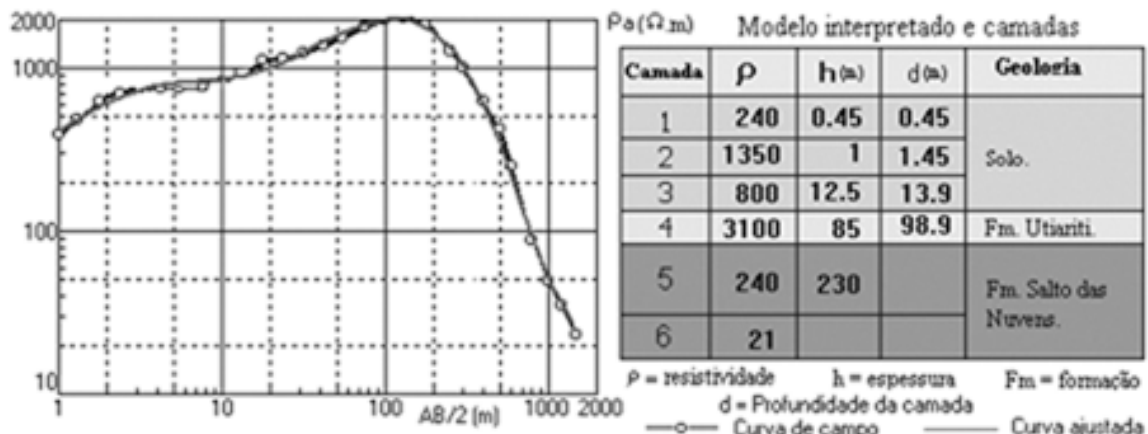

Figura 13 - Perfil geoelétrico da SEV9 e modelo interpretado.

Observa-se que a Formação Utiariti apresenta pequena variação na profundidade ao topo, tendo o seu maior valor no sudoeste da área (Fig. 14) e a sua espessura aumenta suavemente de noroeste para sul (Fig. 15).

A Formação Utiariti, principal aqüífero da área, tem profundidade média em torno de $13 \mathrm{~m}$ e espessura variando de $75 \mathrm{~m}$ a $106 \mathrm{~m}$ em toda área pesquisada. Assim, podem ser construídos poços com profundidade de $50 \mathrm{~m}$ até $120 \mathrm{~m}$ em qualquer parte da área, sendo que poços com $120 \mathrm{~m}$ produzirão maiores vazões com menores rebaixamentos, uma vez que estes penetrarão todo 0 aqüífero (Fetter, 2002; Custódio \& Llamas, 2002). Além disso, a espessura desse aqüífero pode ser usada para estimar as suas reservas de água (Cutrim \& Rebouças, 2005a; Fetter, 2002; Costa, 2000) e para auxiliar na elaboração de projeto de poços tubulares (Custódio \& Llamas, 2002) e na formulação do modelo hidrogeológico conceitual da área. 


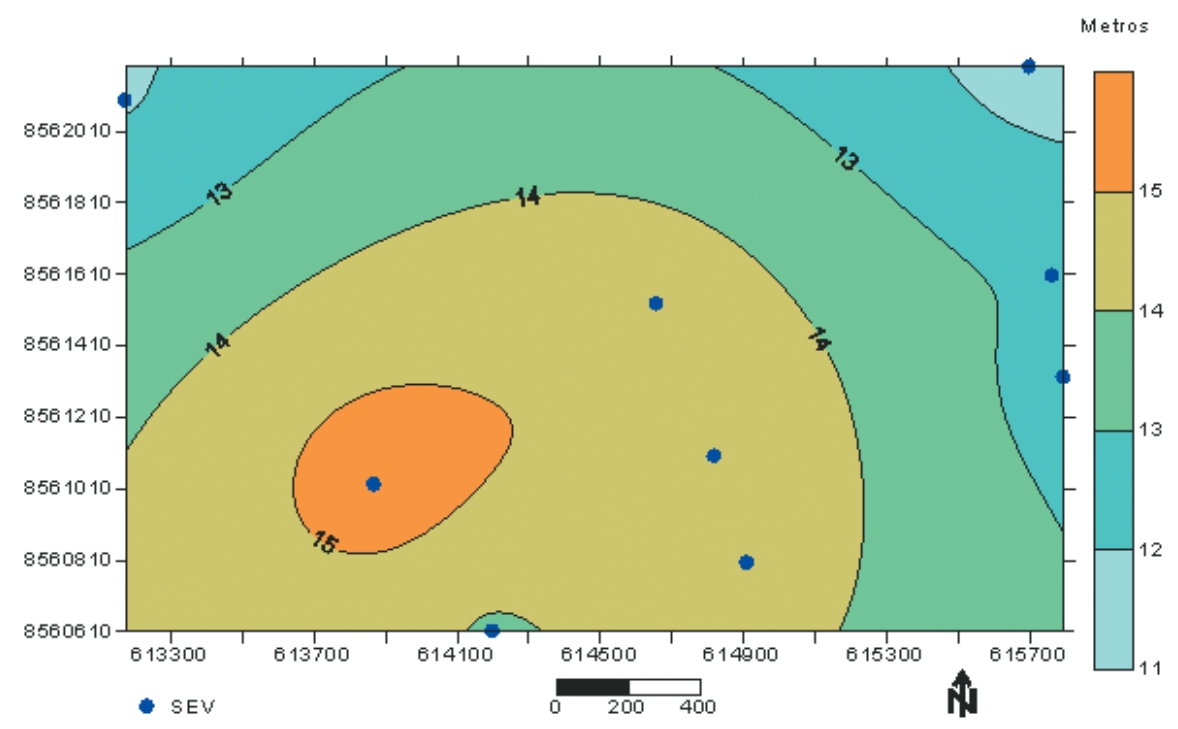

Figura 14 - Mapa de isoprofundidade do Aqüífero Utiariti produzido pelas SEVs.

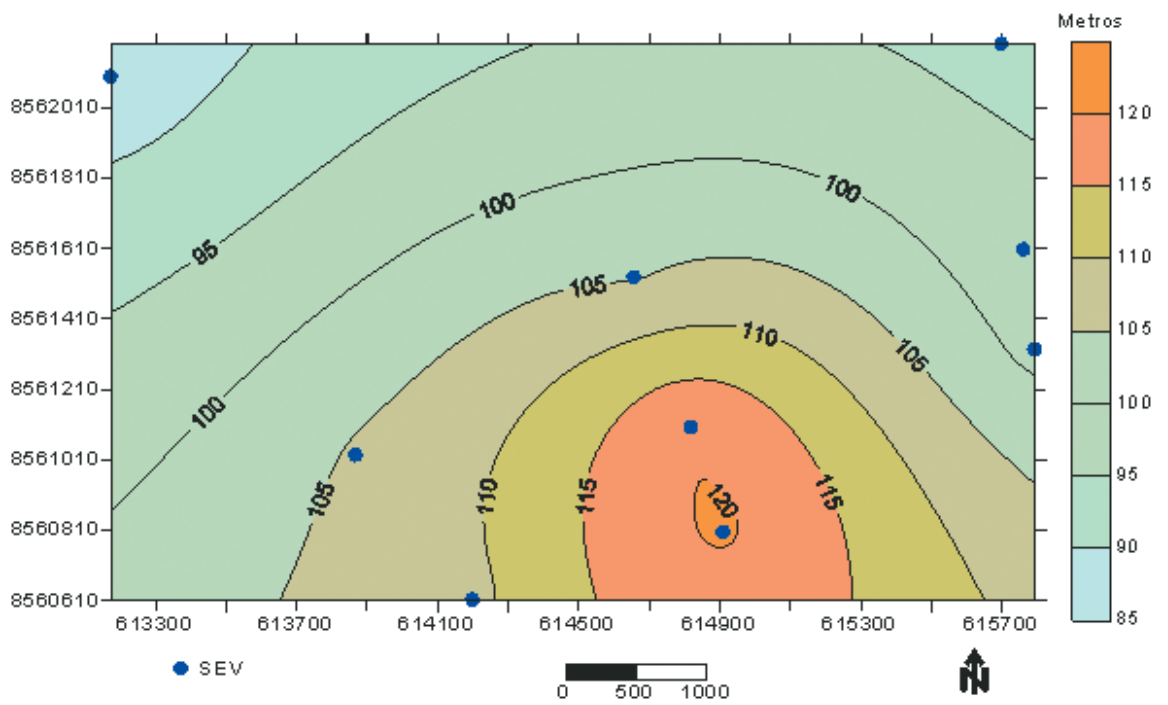

Figura 15 - Mapa de isoespessura do Aqüífero Utiariti produzido pelas SEVs.

\section{CONCLUSÕES E SUGESTÕES}

Os resultados mostram que a Sondagem Elétrica Vertical foi muito eficiente na estimativa da espessura e da profundidade ao topo das unidades geológicas da área, mostrando uma grande homogeneidade na espessura dessas unidades. Destaca-se a definição precisa da porção da Formação Utiariti constituída essencialmente pelos arenitos de textura fina e média, é a parte da formação com maior capacidade para armazenar e liberar água.

No entanto, somente parte da variação textural do arenito da
Formação Utiariti foi evidenciada nas curvas das SEVs, devido, principalmente, a pequena espessura da maioria dessas camadas, comparadas com as aberturas de $A B / 2$ correspondentes às suas profundidades.

Considerando que a Formação Utiariti é o principal aqüífero da área, e tem espessura média de $90 \mathrm{~m}$ e profundidade ao topo em torno de 13 m, então poços para explorar esse aqüífero devem ter profundidade variando de $50 \mathrm{~m}$ a $110 \mathrm{~m}$.

Os dados produzidos nesta pesquisa serão de grande utilidade para estimar as reservas de água do Aqüífero Utiariti, ava- 
Tabela 1 - Espessura e profundidade das unidades geológicas estimadas pelas SEVs.

\begin{tabular}{|l|c|c|c|c|c|c|c|c|c|c|}
\hline \multirow{2}{*}{ Geologia } & \multicolumn{2}{|c|}{ SEV1 } & \multicolumn{2}{c|}{ SEV2 } & \multicolumn{2}{c|}{ SEV3 } & \multicolumn{2}{c|}{ SEV4 } & \multicolumn{2}{c|}{ SEV5 } \\
\cline { 2 - 11 } & $\mathrm{h}$ & $\mathrm{d}$ & $\mathrm{h}$ & $\mathrm{d}$ & $\mathrm{h}$ & $\mathrm{d}$ & $\mathrm{h}$ & $\mathrm{d}$ & $\mathrm{h}$ & $\mathrm{d}$ \\
\hline Sol0 & 13,8 & 0 & 15,6 & 0 & 11,9 & 0 & 14,7 & 0 & 11,5 & 0 \\
\hline Fm. Utiariti & 95 & 13,8 & 90 & 15,6 & 75 & 11,9 & 90 & 14,7 & 82 & 11,5 \\
\hline $\begin{array}{l}\text { Fm. Salto } \\
\text { das Nuvens }\end{array}$ & $>550$ & 108,8 & $>550$ & 105,6 & $>550$ & 86,9 & $>550$ & 104,7 & $>550$ & 93,5 \\
\hline
\end{tabular}

Tabela 1 - continuação

\begin{tabular}{|l|c|c|c|c|c|c|c|c|}
\hline \multirow{2}{*}{ Geologia } & \multicolumn{2}{|c|}{ SEV6 } & \multicolumn{2}{c|}{ SEV7 } & \multicolumn{2}{c|}{ SEV8 } & \multicolumn{2}{c|}{ SEV9 } \\
\cline { 2 - 9 } & $\mathrm{h}$ & $\mathrm{d}$ & $\mathrm{h}$ & $\mathrm{d}$ & $\mathrm{h}$ & $\mathrm{d}$ & $\mathrm{h}$ & $\mathrm{d}$ \\
\hline Sol0 & 14,9 & 0 & 12,9 & 0 & 14,5 & 0 & 13,9 & 0 \\
\hline Fm. Utiariti & 105 & 14,9 & 84 & 12,9 & 106 & 14,5 & 85 & 13,9 \\
\hline $\begin{array}{l}\text { Fm. Salto } \\
\text { das Nuvens }\end{array}$ & $>550$ & 119,9 & $>550$ & 96,9 & $>550$ & 120,5 & $>550$ & 98,9 \\
\hline
\end{tabular}

$F m=$ formação, $h=$ espessura $(m), d=$ profundidade $(m)$.

liar a sua vulnerabilidade à contaminação, formular o seu modelo hidrogeológico conceitual e para elaborar projeto de poços tubulares para explorar esse aqüífero.

\section{REFERÊNCIAS}

ALLER L, BENNETT T, LEHR JH, PETTY R \& HACKETT G. 1987. DRASTIC: A standardized system for evaluating groundwater pollution potential using hydrogeologic settings. USEPA, EPA/600/2-87/035, EUA.

ANDERSON MP \& WOESSNER WW. 1992. Applied groundwater modeling: simulation of flow and advective transport. Academic Press, New York, $381 \mathrm{p}$.

BARROS LC, CARDOSO ORFA, FREIRE FA, SOUZA JÚNIOR JJ, RIVETTI LUZ DS, PALMEIRA RCB \& TASSINARI CCG. 1982. Geologia da folha SD-21. Cuiabá. Projeto RADAMBRASIL, Rio de Janeiro, RJ, 531 p.

BHATTACHARYA PK \& PATRA HP. 1986. Direct Current Geoelectric Sounding. Elsevier Publishing Company, New York, $134 \mathrm{p}$.

COSTA WD. 2000. Uso e gestão de água subterrânea. In: Hidrogeologia conceitos e aplicações. CPRM Serviço Geológico Nacional, 341-365.

CUSTÓDIO E \& LLAMAS MR. 2002. Hidrologia subterrânea. $2^{\text {a }}$ ed., Ediciones Omega, S.A, Barcelona, p. 1165-2350.

CUTRIM AO \& FACHIN SJS. 2004. Aplicação integrada de sondagem elétrica vertical e perfil geológico de poço na determinação da profundidade e da espessura de unidades hidrogeológicas da Bacia do Paraná no município de Poxoréo-MT. XIII Congresso Brasileiro de Águas Subterrâneas. Cuiabá-MT, CD-ROM.
CUTRIM AO \& REBOUÇAS AC. 2005a. Estimativa das reservas de água do Aqüífero Furnas na cidade de Rondonópolis-MT. $1^{\circ}$ Simpósio de Recursos Hídricos do Sul e $1^{\circ}$ Simpósio de Águas da AUGM. Santa Maria-RS, CD-ROM.

CUTRIM AO \& REBOUÇAS AC. 2005b. Aplicação de sondagem elétrica vertical na estimativa do topo e da espessura de unidades geológicas da Bacia do Paraná na cidade de Rondonópolis-MT. Revista Brasileira de Geofísica. Rio de Janeiro, 23(1): 89-98.

CUTRIM A0, SHIRAIWA S, CASARIN JC \& REBOUÇAS AC. 2002. Locação de poços tubulares profundos na Bacia do Paraná, no município de Rondonópolis - MT, usando sondagem elétrica vertical. XII Congresso Brasileiro de Águas Subterrâneas. Florianópolis-SC, CD-ROM.

CUTRIM A0, SHIRAIWA S \& NASCIMENTO AL. 2001. Contribuição da sondagem elétrica vertical à hidrogeologia da Bacia do Paraná no município de Juscimeira-MT. $7^{\circ}$ Congresso Internacional da Sociedade Brasileira de Geofísica, Salvador-BA, CD-ROM.

FETTER CW. 2002. Applied hydrogeology. Prentice-Hall, Inc. 4th ed., $598 \mathrm{p}$.

FOSTER S, HIRATA R, GOMES D, D'ELIA M \& PARIS M. 2002. Groundwater quality protection: a guide for water service companies, municipal authorities and environment agencies. 101 p.

PRODEAGRO. Programa de Desenvolvimento do Agronegócio. 2001. Aspectos geológicos da Folha Rio Claro - MIR 356 (SD.21-X-C). 235 p.

TIKHONOV AN \& ARSENIN VY. 1977. Solutions of ill-posed problems. Winston \& Sons, New York, 349 p. 


\section{NOTAS SOBRE OS AUTORES}

Alterêdo Oliveira Cutrim é graduado em matemática pela Universidade Federal do Maranhão em 1980. Mestrado em Geofísica pelo Centro de Geociências da UFPA em 1985. Doutorado em Ecologia e Conservação da Biodiversidade, IB/UFMT em 1999, com tese em água subterrânea e desenvolvimento urbano sustentável. Professor Adjunto do Departamento de Geologia Geral da Universidade Federal de Mato Grosso, com atuação em Hidrogeologia e Geofísica Aplicada à Hidrogeologia.

Amarildo Salina Ruiz é graduado em geologia pela Universidade Federal de Mato Grosso em 1986. Mestrado em geologia pela Universidade de São Paulo (USP) em 1992. Doutorado em Geociências pela Universidade Estadual Paulista (UNESP - Rio Claro) em 2005. Professor Adjunto do Departamento de Geologia Geral da Universidade Federal de Mato Grosso.

Lucas Matos Liporoni é aluno do Curso de Engenharia Sanitária e Ambiental da Universidade Federal de Mato Grosso - UFMT, Faculdade de Arquitetura e Engenharia - FAET. Bolsista de Iniciação Científica.

Felipe Almeida de Medeiros é aluno do Curso de Geologia da Universidade Federal de Mato Grosso - UFMT, Instituto de Ciências Exatas e da Terra - ICET. Bolsista de Iniciação Científica.

Uendel Chaves Barroso é aluno do Curso de Geologia da Universidade Federal de Mato Grosso - UFMT, Instituto de Ciências Exatas e da Terra - ICET.

Alexandrino Leite Nascimento é técnico em geofísica do Departamento de Geologia Geral da Universidade Federal de Mato Grosso - UFMT, Instituto de Ciências Exatas e da Terra - ICET desde 1980. 